\title{
Correlation Between Local Elastic Heterogeneities and Overall Elastic Properties in Metallic Glasses
}

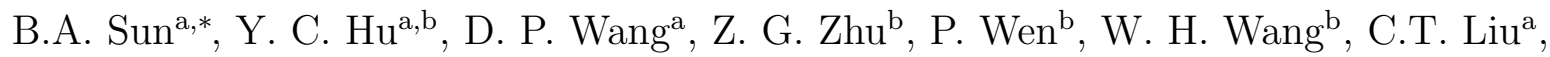 \\ Y. Yang ${ }^{\mathrm{a}, *}$ \\ ${ }^{a}$ Centre for Advanced Structural Materials, Department of Mechanical and Biomedical Engineering, City \\ University of Hong Kong, Kowloon Tang, Kowloon, Hong Kong SAR, China \\ ${ }^{b}$ Institute of Physics, Chinese Academy of Science, 100190, Beijing, China
}

\begin{abstract}
The common notion suggests that metallic glasses (MGs) are a homogeneous solid at the macroscopic scale; however, recent experiments and simulations indicate that MGs contain nano-scale elastic heterogeneities. Despite the fundamental importance of these findings, a quantitative understanding is still lacking for the local elastic heterogeneities intrinsic to MGs. On the basis of Eshelby's theory, here we develop a micromechanical model that correlates the properties of the local elastic heterogeneities, being very difficult to measure experimentally, to the measurable overall elastic properties of MGs, such as shear/bulk modulus and Poisson's ratio. Our theoretical modeling is verified by the experimental data obtained from various MGs annealed to different degrees. Particularly, we revealed that upon annealing, the Poisson's ratio decreases if shear softening dominates the behavior of local soft regions or increase if pressure softening becomes dominant in MGs. Our results provide quantitative insights into the structural mechanism of the Poisson's ratio criterion on the ductility long established for MGs. Finally, The correlation between the Poisson's ratio of a glass and the fragility of its corresponding liquid is also discussed.
\end{abstract}

Keywords: Metallic glass, Elastic properties, Structural heterogeneities, Ductility

\footnotetext{
*Corresponding author

Email addresses: iphysunba@gmail.com (B.A. Sun), yonyang@cityu.edu.hk (Y. Yang)
} 


\section{Introduction}

Despite the fundamental and technological importance, the structure-property relationship in metallic glasses (MGs) has long been obscured by their long-range disordered structure[13]. A MG often looks microstructurally featureless or "amorphous" under a conventional electron microscope, which is in sharp contrast to crystalline alloys in which grain boundaries, dislocations and many other microstructural features can be readily identified[2]. However, recent atomistic simulations [4-8]and experiments[9-13] indicate that the amorphous structure of glasses is indeed heterogeneous, containing nano-scale elastic[10, 12] or viscoelastic $[11,13]$ inhomogeneities on top of their intrinsic density fluctuation. Unlike the conventional microstructural defects in crystalline alloys, the structural heterogeneities in MGs are dynamic in nature, which are invisible under static electron microscopes but could be theoretically associated with low-frequency vibration modes[7, 14, 15] or liquid-like regions with very low local viscosities or relaxation times[13, 16]. In principle, these regions of dynamic heterogeneity are susceptible to irreversible local atomic rearrangements under external perturbations (stress or heat), thus acting as "flow units" to initiate macroscopic plastic flows[17, 18] or as "liquid-like sites" to initiate secondary relaxations in MGs[19, 20]. Despite the recent efforts confirming the existence of the dynamic heterogeneities in MGs, however, it still remains challenging to derive the mechanical/physical properties of MGs, in a quantitative manner, from the perspective of local structural heterogeneities.

On the other hand, through the extensive studies over the past decades[21, 22], various correlations were established between the overall elastic constants of MGs and their many other physical properties. Today, it is commonly thought that the elastic properties of MGs may hold the key to the understanding of many fundamental issues in MGs, such as glass transition[23-25], relaxation[26, 27] and deformation[28, 29]. For example, it was shown that the elastic modulus of a MG is determined by both the atomic bonding strength and the atomic configuration[27, 30], which varies with the possible structural change in a MG, as witnessed in a typical structural relaxation[28, 31, 32] or rejuvenation process[33, 34]. Moreover, extensive studies also indicate that the Poisson's ratio of a MG correlates with 
the structural state of the corresponding supercooled liquid, as characterized by the liquid fragility[24], which in turn affects the ductility of the glass. As motivated by the fact that the amorphous structure of MGs is overall heterogeneous, we intend to derive relations in this work that connect the elastic constants of MGs to their local structural heterogeneities. Once these relations become available, one may develop important structure-property relations, with the use of the elastic constants as intermediate variables, which ultimately bridge the structural heterogeneity in MGs and their physical and mechanical properties or other attributes.

\section{Theoretical Modelling}

In principle, the whole structure of an MG can be envisaged as an atomic-scale composite[13, 35], consisting of the solid-like regions (SLRs) as a "matrix" and isolated liquid-like regions (LLRs) as "inclusions", as schematically shown in Figure 1. The LLRs are loosely packed atom regions, and should have the lower elastic moduli (bulk or shear modulus) than those of rigid SLRs. From the mechanistic point of view, LLRs can be viewed as elastic inhomogeneities embedded in an isotropic matrix. Thus, the effective elastic moduli of the whole structure can be readily obtained from Eshelby's theory[36], which is a continuum approach in elasticity and take into account elastic mismatch caused by the inelastic transformation of an inclusion or the presence of an inhomogeneity in a isotropic or anisotropic elastic matrix. In amorphous solids, Eshelby's theory has been extensively used in analyzing the elastic stress field associated with the shear transformation of elementary deformation units[37-39], which are possibly initiated from LLRs. Here, we consider the static or "unrelaxed" elastic modulus of MGs for study. Suppose that the composite structure as shown in Fig.1 is subject to a uniform applied elastic strain field of $\epsilon_{i j}^{A}(i, j$ over $1,2,3)$, the elastic mismatch between the inhomogeneities and the matrix will cause an additional stress/strain field, leading to an interaction elastic energy. The effective elastic modulus $\left(C_{i j k l}\right)$ is defined from the total elastic energy in the specimen:

$$
E_{\text {total }}=E_{0}+\sum E_{i n t}=\frac{1}{2} C_{i j k l} \epsilon_{i j}^{A} \epsilon_{k l}^{A}
$$


In Eq.1, $E_{0}$ is the elastic energy of the specimen under a uniform $\epsilon_{i j}^{A}$ when it is free of inhomogeneities. $E_{i n t}$ is the interaction energy between an inhomogeneity and the external field. The sum is over all inhomogneities contained in the matrix. Both $E_{0}$ and $E_{\text {int }}$ are the quadratic functions of $\epsilon_{i j}^{A}$. Assume the bulk (or shear) modulus of SLRs and LLRs are $K_{0}$ (or $G_{0}$ ) and $K_{1}$ (or $G_{1}$ ), respectively, the effective bulk $(K)$ or shear $(G)$ modulus of the SSRs-LLRs assembly is derived as (See Appendix A for detailed derivation):

$$
K=\frac{K_{0}}{1+A V_{f}}, G=\frac{G_{0}}{1+B V_{f}}
$$

where $V_{f}$ is the volume fraction of LLRs. $A$ and $B$ are two coefficients associated with the discrepancy of elastic moduli between SLRs and LLRs. It should be noted here that the elastic moduli of LLRs in MGs are not uniform and might have a wide distribution; however, the current theoretical approach is still valid in a mean-field sense if $K_{1}$ (or $G_{1}$ ) is viewed as the mean value of bulk (or shear) modulus of LLRs dispersed in MGs. For spherical LLRs, $A$ and $B$ read[36]:

$$
A=\frac{K_{1}-K_{0}}{\left(K_{0}-K_{1}\right) \alpha-K_{0}}, B=\frac{G_{1}-G_{0}}{\left(G_{0}-G_{1}\right) \beta-G_{0}}
$$

where the parameters $\alpha$ and $\beta$ are functions of the Poisson's ratio of the matrix, $\nu_{0}: \alpha=$ $(1 / 3)\left(1+\nu_{0}\right) /\left(1-\nu_{0}\right), \beta=(2 / 15)\left(4-5 \nu_{0}\right) /\left(1-\nu_{0}\right) . A$ and $B$ usually have positive values due to the elastic softening of LLRs, as compared to SLRs $\left(K_{1}<K_{0}, G_{1}<G_{0}\right)$. Finally, the effective Poisson's ratio of MGs can be derived from Eq.2 based on the constitutive relations of elastic constants in an isotropic solid:

$$
\nu=\frac{\left(1+\nu_{0}\right)\left(1+B V_{f}\right)-\left(1-2 \nu_{0}\right)\left(1+A V_{f}\right)}{2\left(1+\nu_{0}\right)\left(1+B V_{f}\right)+\left(1-2 \nu_{0}\right)\left(1+A V_{f}\right)}
$$

where $\nu_{0}=\left(3 K_{0}-2 G_{0}\right) /\left(6 K_{0}+2 G_{0}\right)$. It should be noted that the Eshelby approach can be also extended to study other types of elastic inhomogeneities (e.g. pre-existing shear bands,) which can be viewed as the planar soft elastic inhomogeneities in a glassy matrix. In these cases, the expressions of $\alpha$ and $\beta$ with $\nu_{0}$ have to be modified because of the shape anisotropy of elastic inhomogeneities.

Eqs. 2-4 establish general relations between the macroscopic elastic properties and the characteristics of microscopic structural heterogeneities in MGs. From these relations, one 


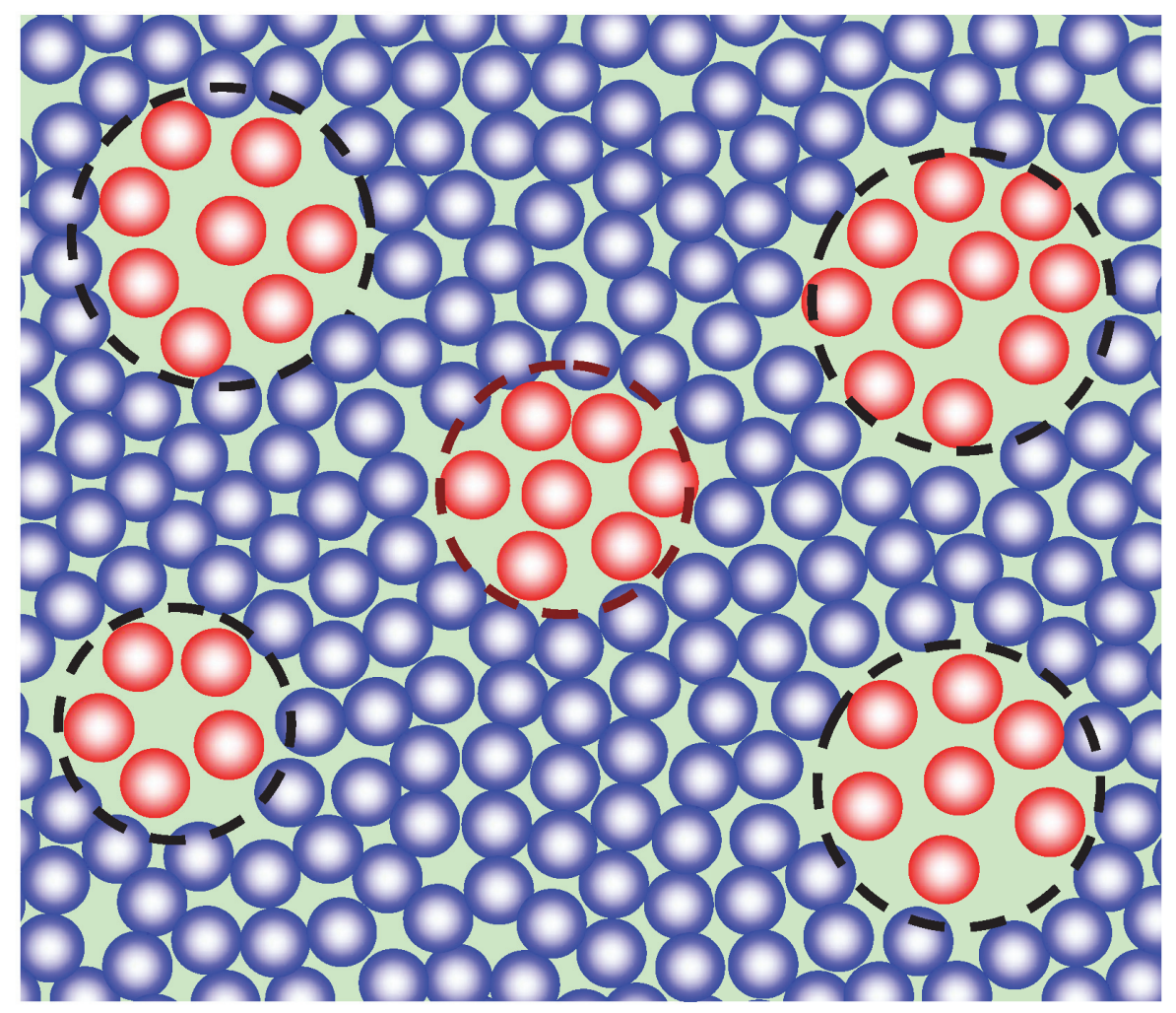

Figure 1: The schematic for the atomic-scale structural heterogeneities in MGs. The loosely packed region (in red atoms) are embedded in the matrix of densely-packed region (in blue atoms). 
can generally find that the overall elastic moduli of MGs do not follow the simple "rule of mixtures", i.e. the weighted average of elastic moduli of SLRs and LLRs, which commonly used in the previous studies to estimate the elastic properties of MGs[21]. To facilitate discussion, we define $\xi=\left(K_{0}-K_{1}\right) / K_{0}$ and $\psi=\left(G_{0}-G_{1}\right) / G_{0}$, which reflect the degree of elastic softening in LLR relative to SLRs. In general, $\xi$ and $\psi$ range from 0 to 1 . In limiting case of $\xi=1$ and $\psi=1$, LLRs behave like cavitation, while for $\xi<1$ and $\psi=1$, LLRs behave like normal liquids with a Poisson's ratio approaching 0.5[40].

According to Eq. 3, $A$ and $B$ can be expressed as a function of $\xi$ and $\psi$, respectively: $A=\xi /(1-\alpha \xi), B=\psi /(1-\beta \psi)$. Therefore, the elastic moduli are intrinsically correlated with $\xi$ and $\psi$ according to Eq. 2 and Eq.4. Figure 2 shows the variation of overall elastic moduli with the volume fraction of SLRs at different values of $\xi$ and $\psi$. As seen in this figure, both $K$ and $G$ decrease monotonically with $V_{f}$, and their decreasing rate depend on the specific value of $\xi$ or $\psi$ : the larger is the $\xi$ or $\psi$ is, the more steeply does the $K$ or $G$ drop. In contrast, the Poisson's ratio could either decrease or increase with $V_{f}$, depending on the specific values of $\xi$ and $\psi$. Based on this phenomenon, one can define a shear-softening dominated region and a pressure-softening dominated region, as shown in Fig 2c. In the shear-softening dominated region, $\xi<\psi$ and $\nu$ increase monotonically with $V_{f}$. This behavior agrees with the common notion that introducing more LLRs into a glassy structure would lead to a higher Poisson's ratio, thus promoting plasticity in MGs[19, 41]. In contrast, in the pressure-softening region, $\xi \geq \psi$ and $\nu$ decrease monotonically with $V_{f}$. This behavior is somewhat opposite to our common knowledge and has never been reported before for MGs. In what follows, we will provide experimental evidences to validate our theory. To highlight the boundary between shear-softening and pressure-softening regions, Fig. 2d shows a contour plot of the effective Poisson's ratio as a function of $\xi$ and $\psi$ at a volume fraction, $V_{f}=30 \%$. From the plot, one can see that the two regions meet at the boundary that corresponds to $\xi=\psi$. In the region $\xi<\psi$ (shear softening), the effective or overall Poisson's ratio is higher than that of SLRs, $\nu_{0}$, while in the region $\xi>\psi$ (pressure softening), the effective Poisson's ratio is lower than $\nu_{0}$. The magnitude of Poisson's ratio varies with $\xi$ and $\psi$. Depending on the value of $\nu_{0}, \nu$ could even reach a negative value 
at a large $V_{f}$ and a large discrepancy between $\xi a n d \psi$, resulting a negative Poisson's ratio as reported before in materials[40]. In principle, the Eshelby approach can be also used to model the case of local soft regions with negative Poisson's ratio, i.e. $3 K_{1}<2 G_{1}$. This will result in relatively large $\xi$ and relatively small $\psi$, tuning the behavior of local soft regions towards the pressure-softening dominated regime.

\section{Experimental Methods and Results}

\subsection{Experimental Methods}

MG alloy ingots with the nominal compositions $\mathrm{Zr}_{52.5} \mathrm{Cu}_{17.9} \mathrm{Al}_{10} \mathrm{Ni}_{14.6} \mathrm{Ti}_{5}(\mathrm{Vit105}), \mathrm{Zr}_{41} \mathrm{Ti}_{14}$ $\mathrm{Cu}_{12.5} \mathrm{Ni}_{10} \mathrm{Be}_{22.5}$ (Vit1), $\mathrm{Zr}_{46.75} \mathrm{Ti}_{8.25} \mathrm{Cu}_{7.5} \mathrm{Ni}_{10} \mathrm{Be}_{27.5}$ (Vit4) and $\mathrm{Ce}_{68} \mathrm{Al}_{10} \mathrm{Cu}_{20} \mathrm{Fe}_{2}$ and $\mathrm{La}_{60} \mathrm{Ni}_{15} \mathrm{Al}_{25}$ were produced by arc melting a mixture of pure metals (purity $\geq 99.5 \%$ in mass weight) in a Ti-gettered argon atmosphere. Glassy alloy rods with diameters of 2-10 $\mathrm{mm}$ and a length of at least $30 \mathrm{~mm}$ were obtained by suction casting into a water-cooling copper mould. The amorphous nature of specimens both at the as-cast state and after thermal annealing were confirmed by the x-ray diffraction (XRD) method using a MAC Mo3 XHF diffractometer with $\mathrm{Cu} \mathrm{K} \alpha$ radiation and the differential scanning calorimetry (DSC, Perkin Elmer DSC7). Thermal annealing experiments were performed in a Muffle furnace. The MG samples were first encapsulated into a quartz tube with a vacuum of $10^{-4} \mathrm{~Pa}$ and then annealed at their sub- $T_{g}$ temperatures for different times. The annealing temperature for Vit105, Vit1, Vit4, the Ce- and La-based MGs was set to be $600 \mathrm{~K}, 623 \mathrm{~K}, 523 \mathrm{~K}, 333 \mathrm{~K}$ and $423 \mathrm{~K}$, respectively. After a certain annealing time, the glassy sample was removed from the furnace and cooled to room temperature for elastic modulus measurement. Then the same sample was encapsulated and put back to the furnace for further annealing. This process was repeated until all elastic measurements are completed at different annealing times.

The elastic moduli were measured at room temperature by using a pulse echo overlap method [21, 42]with a MATEC 6600 ultrasonic system. The frequency of the ultrasonic wave was $10 \mathrm{MHz}$. The acoustic longitudinal velocity, $v_{l}$, and shear velocity, $v_{s}$, of MG samples were measured at room temperature The density $\rho$ was measured by Archimedes' 

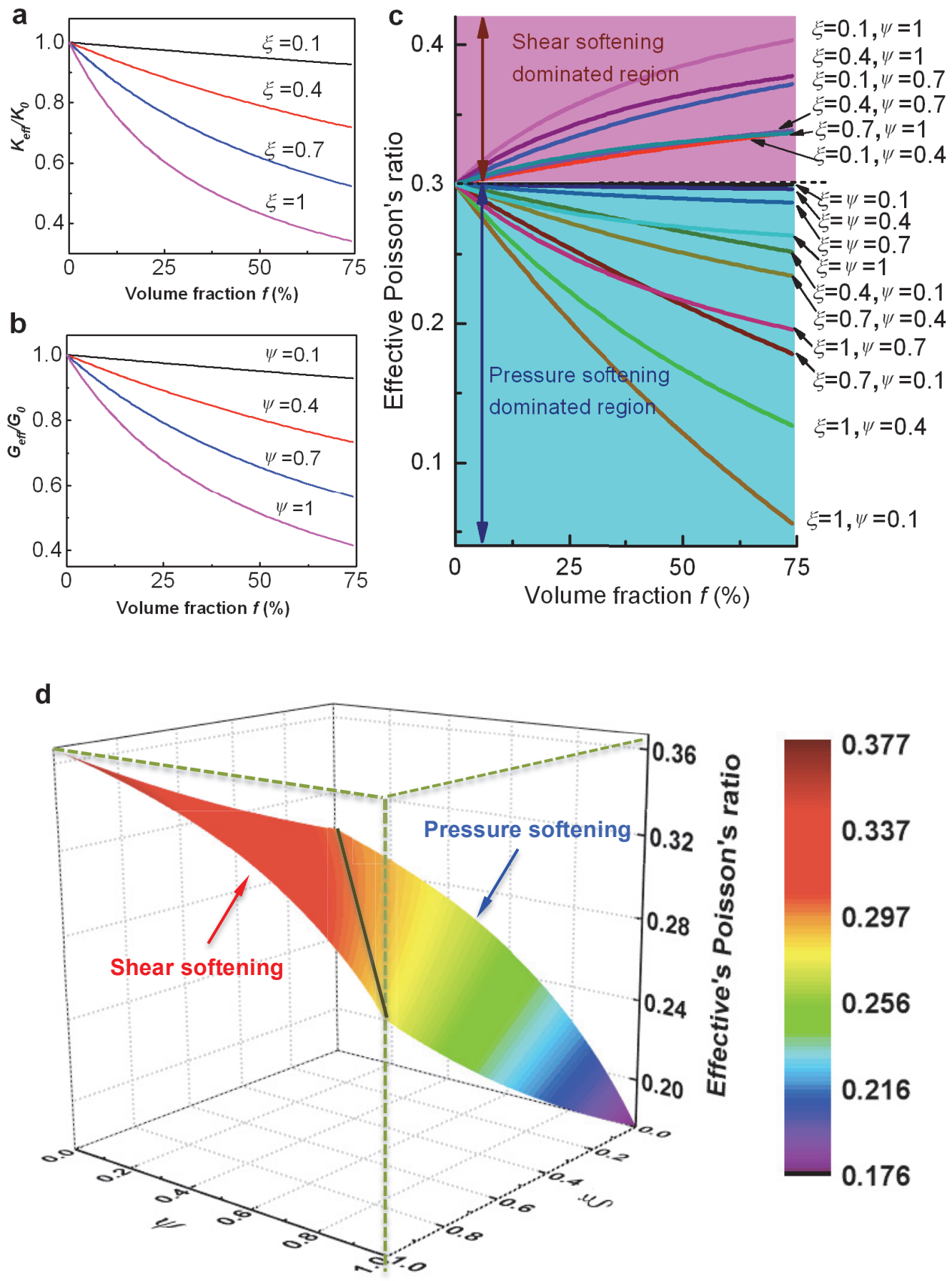

Figure 2: The calculated variation of effective elastic constants with the volume fraction of LLRs for different values of $\xi$ and $\psi$ : (a) the effective bulk modulus $K$; (b) the effective shear modulus $G$; (c) the effective Poisson's ratio $\nu$. (d) The contour plot of the variation of effective Poisson's ratio with $\xi$ and $\psi$ at a typical volume fraction $\left(V_{f}=30 \%\right)$. 
principle in distilled water in an accuracy of $0.5 \%$. The elastic constants, e.g. bulk modulus $K$, shear modulus $G$, Young's modulus $E$ and Poisson's ratio of MGs were derived from the acoustic velocities[21]. The uniaxial compression tests were performed on an Instron 5869 electromechanical test system under a constant strain rate $5 \times 10^{-4} \mathrm{~s}^{-1}$. The load, displacement and the time were recorded at a frequency of $50 \mathrm{~Hz}$. The strain was measured by a laser extensometer(Fiedler) attached to the testing machine.

MD simulations for five MGs, i.e. $\mathrm{Cu}_{50} \mathrm{Zr}_{50}, \mathrm{Cu}_{46} \mathrm{Zr}_{46} \mathrm{Al}_{8}, \mathrm{Mg}_{65} \mathrm{Cu}_{25} \mathrm{Y}_{10}, \mathrm{Ni}_{33} \mathrm{Zr}_{67}, \mathrm{Pd}_{82} \mathrm{Si}_{18}$, were performed using the open-source codes of "Large-scale Atomic/Molecular Massively Parallel Simulator" (LAMMPS). The embedded-atom method (EAM) potentials were employed to describe the interatomic interactions[8]. For each model, the periodic boundary conditions was applied in three dimensions. The initial configuration containing 16,000 atoms was first equilibrated at $2000 \mathrm{~K}$ for $1.5 \mathrm{~ns}$, followed by rapid quenching $\left(10^{12} \mathrm{~K} / \mathrm{s}\right)$ to $300 \mathrm{~K}$ in NPT (constant number, constant pressure and constant temperature) ensemble. To extract the bulk moduli of metallic glasses at various temperatures, an equal deformation strain was applied along the three axes of the simulation box, resulting in a volumetric change. The corresponding pressure $p$ was measured. The bulk modulus was determined from the slope of pressure-dilation strain plots. In all of the simulations, the time step used to integrate the equations of motion was set to be 0.001 ps.

\subsection{The variation of elastic moduli with time during structural relaxation}

The MGs were annealed under the sub- $T_{g}$ temperatures for various times and their elastic properties was measured by a pulse echo overlap ultrasonic method. After long-time annealing, no crystallization took place and the sample remained fully amorphous. During annealing, it can be expected that the volume fraction of LLRs in the glassy structure generally decreases with time as a result of the structural relaxation while the chemical composition of MGs remain unchanged[43]. Therefore, the correlation between elastic properties and microscopic structural heterogeneities in MGs can be explored. Figure 3 shows the change of the measured elastic constants $(K, G, E, \nu)$ with the annealing time $t$ for a typical Vit105 MG. In accordance with previous studies, one can see that $K, G$ and $E$ 
increase sharply at the initial stage and then gradually approach a plateau value for a long annealing, meanwhile, $\nu$ decrease monotonically with the annealing time. In practice, The $K$ or $G$ can be well fitted by the following empirical relation:

$$
P=\frac{P_{\infty}}{1+c_{0} \exp \left(-k t^{n}\right)}
$$

where $P$ can be either $K$ or $G . P_{\infty}, k, n$ are all constant parameters. $E$ and $\nu$ are not fitted here since they can be obtained from $K$ and $G$. The variation of the measured elastic constant with the annealing time for other MGs are displayed in Fig. 4. As can be seen, the variation of $K$ or $G$ with time for other MGs displays a similar trends as Vit105, and can be fitted to Eq.5. However, the obtained overall or effective Poisson's ratio exhibits a composition-dependent behavior, which decrease for Zr-based MGs or increase for Ceand La-based MGs with the annealing time, depending on the MG composition. This is in general accordance with our theoretical analysis in Section 2. The fitting results for all MGs are listed in Table 1. One can see that the exponent $n$ ranges from 0 to 1 , depending on the MG composition. The $K_{\infty}$ and $G_{\infty}$ denote the modulus obtained upon infinite-time annealing, and can be regarded equivalent to those of SLRs, $K_{0}$ and $G_{0}$, respectively. Here, it is worth noting that, when the term $c_{0} \exp \left(-k t^{n}\right)$ is much smaller than 1, Eq.5 is reduced to $P_{\infty}\left[1-c_{0} \exp \left(-k t^{n}\right)\right]$ as a first-order approximation, which is in good agreement with the formula used to describe the relaxation kinetics in different kinds of glasses as reported in the previous studies[44, 45].

\subsection{Elastic modulus softening in LLRs}

In general, it is difficult to directly measure the volume fraction of LLRs, $V_{f}$, in MGs. However, we noticed from Eq. 2 that $V_{f}$ is related to the measured shear modulus upon annealing through the relation: $V_{f}=\left(G_{0} / G-1\right) / B$. Substituting this relation into Eq.5, we obtain:

$$
\nu=\frac{\left(1+\nu_{0}\right)\left(G_{0} / G\right)-\left(1-2 \nu_{0}\right)\left[1+(A / B)\left(G_{0} / G-1\right)\right]}{2\left(1+\nu_{0}\right)\left(G_{0} / G\right)-\left(1-2 \nu_{0}\right)\left[1+(A / B)\left(G_{0} / G-1\right)\right]}
$$

Since $G_{0}$ and $\nu_{0}$ can be obtained through fitting Eq.5 the modulus data, the ratio $A / B$ is the only adjustable parameter here. We plot the measured $\nu$ versus $\left(G_{0} / G-1\right)$ for different 

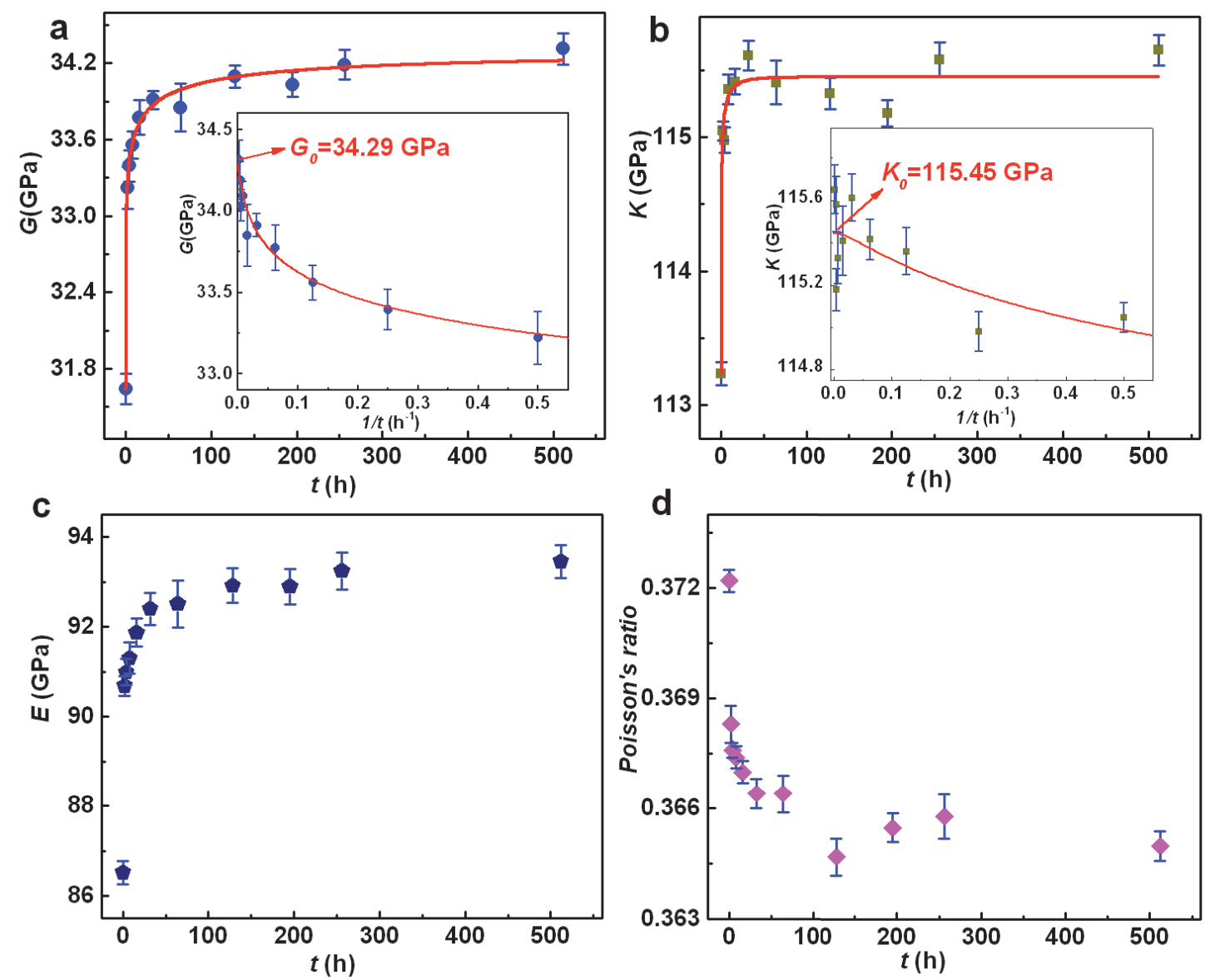

Figure 3: The change of measured elastic constants with the annealing time $t$, respectively for Vit105 MG: (a) bulk modulus $K$; (b) shear modulus $G$; (c) Young's modulus $E$; (d) Poisson's ratio $\nu$. The inset shows variations of $K$ and $G$ with the inverse time, so $K_{0}$ and $G_{0}$ can be easily identified.

Table 1: The values of various parameters obtained by fitting the elastic modulus data during structural relaxation (bulk modulus $K_{0}$, shear modulus $G_{0}$, Poisson's ratio $\nu_{0}$ of elastic matrix, the exponent $n$ and the parameter $k$ for various MGs).

\begin{tabular}{lccccc}
\hline MGs & $K_{0}(\mathrm{GPa})$ & $G_{0}(\mathrm{GPa})$ & $\nu_{0}$ & $n$ & $k$ \\
\hline Vit105 & 115.48 & 34.29 & 0.365 & 0.24 & 0.831 \\
Vit1 & 115.42 & 43.56 & 0.332 & 0.72 & 0.331 \\
Vit4 & 111.83 & 37.10 & 0.351 & 0.37 & 0.416 \\
$\mathrm{Ce}_{68} \mathrm{Al}_{10} \mathrm{Cu}_{20} \mathrm{Fe}_{2}$ & 32.20 & 11.80 & 0.337 & 0.98 & 0.031 \\
$\mathrm{La}_{60} \mathrm{Ni}_{15} \mathrm{Al}_{25}$ & 47.35 & 15.69 & 0.351 & 0.47 & 0.496 \\
\hline
\end{tabular}



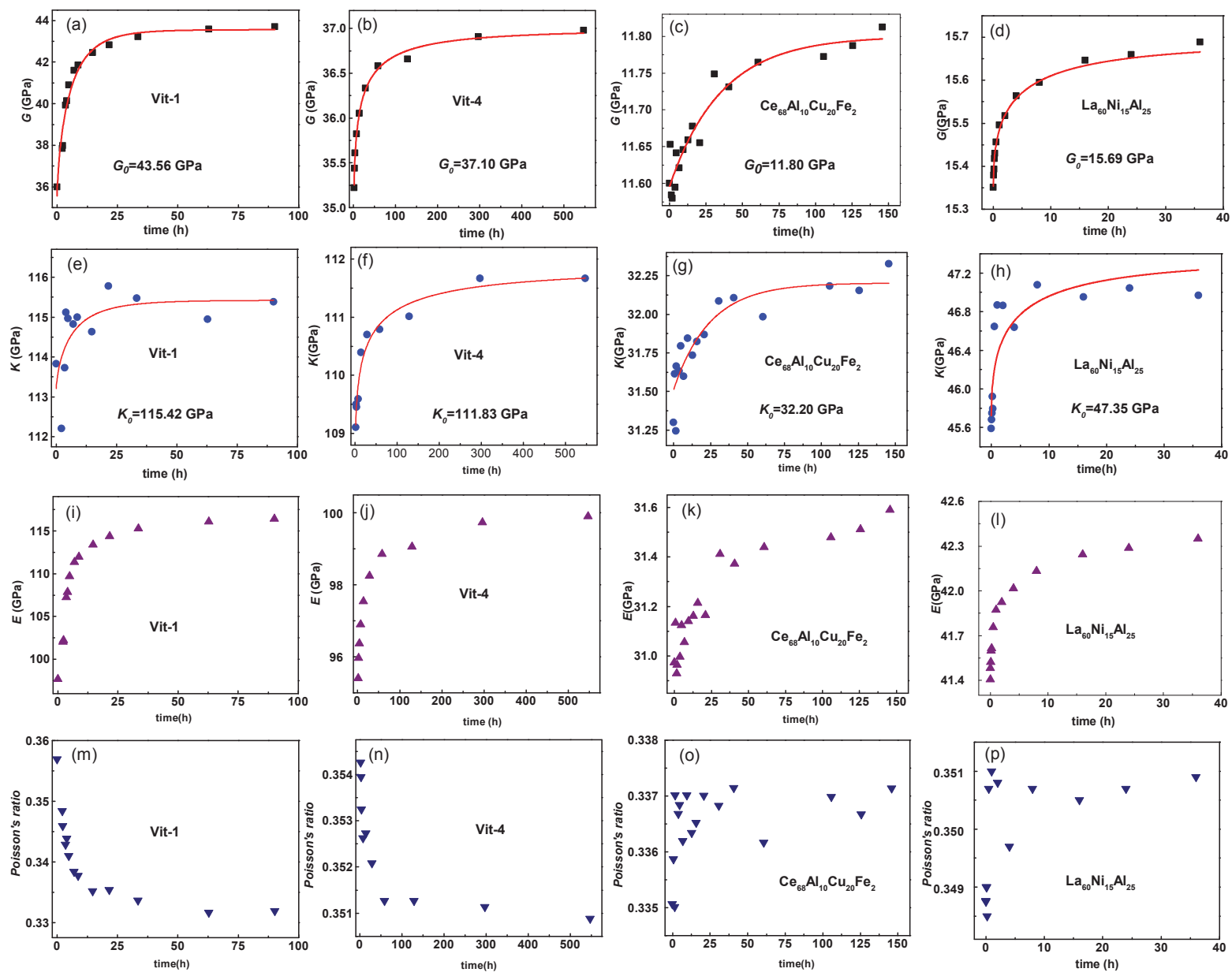

Figure 4: The variations of elastic properties with annealing time during structural relaxation for different MGs (Vit-1, Vit4, $\mathrm{Ce}_{68} \mathrm{Al}_{10} \mathrm{Cu}_{2} 0 \mathrm{Fe}_{2}$ ): (a)-(c) shear modulus $G$; (d)-(f) bulk modulus $K$; (g)-(i) Young's modulus E; (j)-(l) Poisson's ratio $\nu$. The shear modulus and bulk modulus are fitted by Eq. 5 and the values of fitting parameters are listed in Table 1. 
MGs, as shown in Figure 5. Clearly, the experimental data can be well fitted to Eq.6, thus verifying the validity of our theoretical model. The fitted values of $A / B$ by fitting are also shown in Figure 5, which are compositional dependent and listed in Table 2. For Zr-based MGs (Vit105, Vit1 and Vit4), the ratio $A / B$ is less than 1, and for the Ce- and La-based MGs, $A / B$ has a value larger than 1 . As can be inferred from Eq.3, the ratio $A / B$ reflects the relative magnitude of $\xi$ to $\psi$, or the relative degree of pressure to shear softening in LLRs. For $A / B<1$ as displayed by Zr-based MGs, we usually have $\xi<\psi$, or in other words, shear-softening is dominated in LLRs, resulting in the increase of $\nu$ with $V_{f}$. For $A / B>1$, pressure-softening becomes dominant in LLRs, which leads to the decrease of $\nu$ with $V_{f}$ as displayed by the Ce- and La-based MGs. These results are in excellent agreement with our theoretical analysis.

It should be noted that the value of $A / B$ alone could not determine the exact degree of softening, whether it stems from pressure or shear, in LLRs. However, we could give a rough estimate if LLRs are considered to be of the supercooled liquid state, or as the "residual liquidity" in MGs[10]. According to the previous studies[21], the relative change of bulk modulus is small ( $<10 \%$ in general) compared to that of shear modulus in MGs, even when the temperature increases from room temperature to the supercooled liquid regime. To validate this, we also performed molecular simulations for five MGs with different potential functions and calculated their bulk modulus change when the temperature increases from 300 $\mathrm{K}$ to temperatures well above their $T_{g}$, as shown in Figure 6. Strikingly, we found that the relative change of bulk modulus for all MGs falls into a narrow range of 5-8\%. Given these results, we choose $\xi=10 \%$. Together with the fitted value of $A / B$, we can now calculate the shear softening parameter, $\psi$, for LLRs in different MGs, as listed in Table 2. One can see that $\psi$ largely varies with the MG composition and seems to positively correlate with the plasticity, ranging from $45 \%$ for plastic Vit105 $\left(\epsilon_{p} \sim 5-6 \%, \epsilon_{p}\right.$ is the final plastic strain) to $18 \%$ for the less plastic Vit4 $\left(\epsilon_{p} \sim 1.5 \%\right)$ and $6.6 \%$ for the completely brittle Ce-based MG (See Figure 7). The correlation between and the plasticity can be understood from the fact that LLRs with relative large shear modulus softening are more susceptible to inelastic deformation under a low shear stress, thus promoting shear band formation at different 

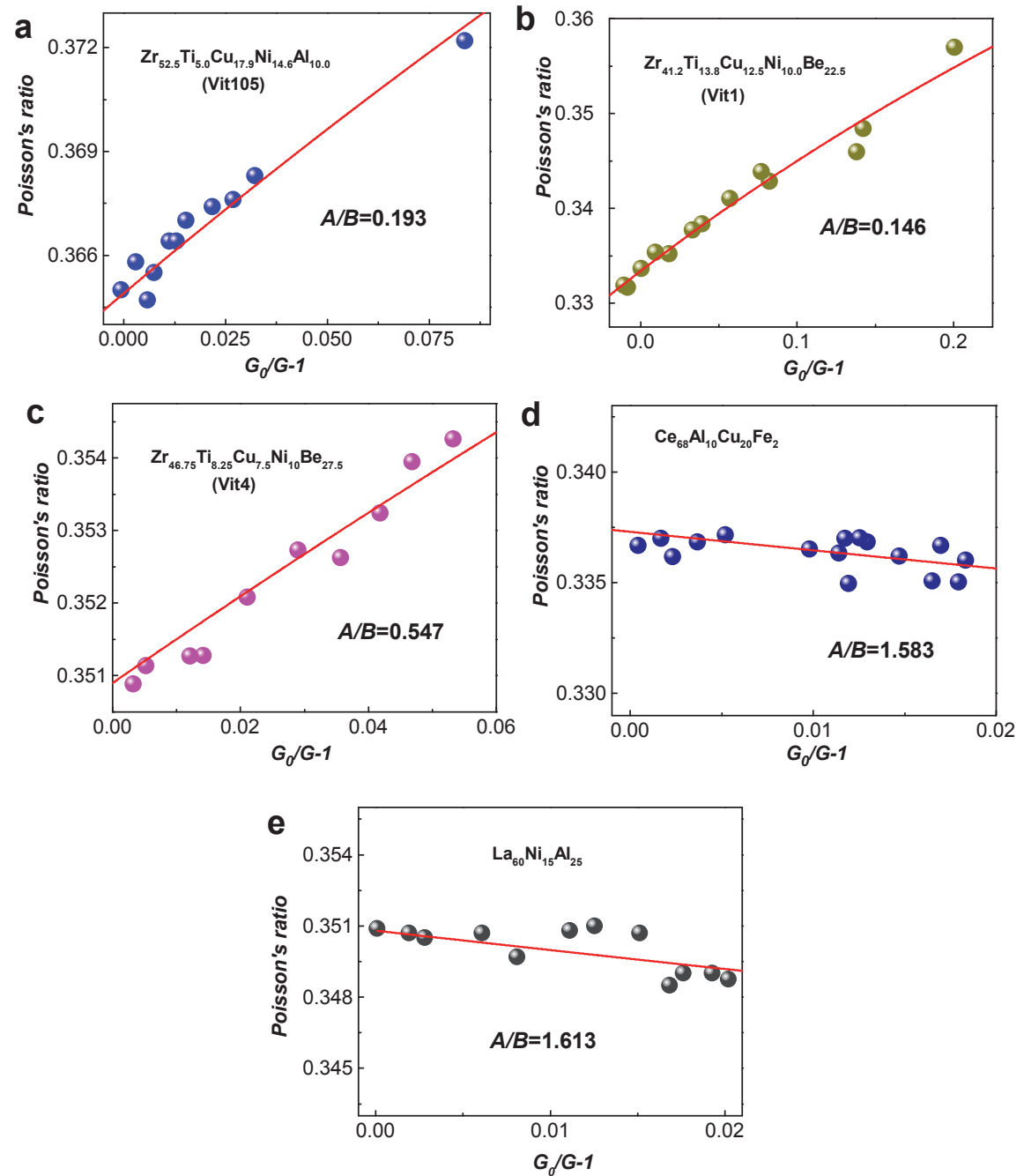

Figure 5: The plot of measured Poisson's ratio, $\nu$, versus $\left(G_{0} / G-1\right)$ upon annealing for different MGs: (a) Vit105; (b) Vit1; (c) Vit4; (d) $\mathrm{Ce}_{68} \mathrm{Al}_{10} \mathrm{Cu}_{20} \mathrm{Fe}_{2}$. All data were well fitted by Eq.6, with the fitting values $A / B$ listed. 
Table 2: The values of various parameters obtained by fitting the elastic modulus data during structural relaxation and molecular dynamic simulations $(A / B, \psi / \xi, \psi)$, and the plastic strain $\epsilon_{p}$ for various MGs.

\begin{tabular}{lcccc}
\hline MGs & $A / B$ & $\psi / \xi$ & $\psi(\%)$ & $\epsilon_{p}(\%)$ \\
\hline Vit105 & 0.193 & 4.5 & 45 & 5.5 \\
Vit1 & 0.146 & 5.4 & 54 & 3.3 \\
Vit4 & 0.547 & 1.8 & 18 & 1.2 \\
$\mathrm{Ce}_{68} \mathrm{Al}_{10} \mathrm{Cu}_{20} \mathrm{Fe}_{2}$ & 1.583 & 0.66 & 6.6 & 0 \\
$\mathrm{La}_{60} \mathrm{Ni}_{15} \mathrm{Al}_{25}$ & 1.613 & 0.64 & 6.4 & 0 \\
\hline
\end{tabular}

sites and giving rise to a greater potential for large plastic deformation. Here it should be emphasized that, within the framework of Eshelby's theory, the correlation between elastic constants and plasticity in MGs, if any, should be valid only up to the yielding point; the post-yielding plastic flow in MGs is governed more by the behavior of shear band sliding, such as the stick-slip dynamics [46-50], which is not taken into account in the current theory. However, the recent work of Sun et al. indicates that instability of shear band sliding is also correlated with the Poisson's ratio of MGs[51]. Therefore, it seems that the correlation between shear-banding mediated plasticity and Poisson's ratio of MGs as proposed by Greer et al.[28, 52] can be justified. Nevertheless, different from the early work[28, 52], one can infer from the current study that this correlation is not solely based on the magnitude of the Poisson's ratio but also depends on how LLRs softens. In the case of pressure softening, the MGs exhibit a brittle behavior even possess a relatively large Poisson's ratio, as seen in Table 2.

\section{Discussion}

Since LLRs are potential "flow units" responsible for macroscopic plastic flow and relaxation behavior in MGs, it is important to investigate their evolutions dynamics with time, which is useful for us to tailor and design the elastic or other properties in MGs. Based on the theoretical analysis and experimental results above, one can easily show that the evolution of the LLR volume fraction with annealing time fits into the form: $V_{f}=V_{f 0} \exp \left(-k t^{n}\right)$, where 

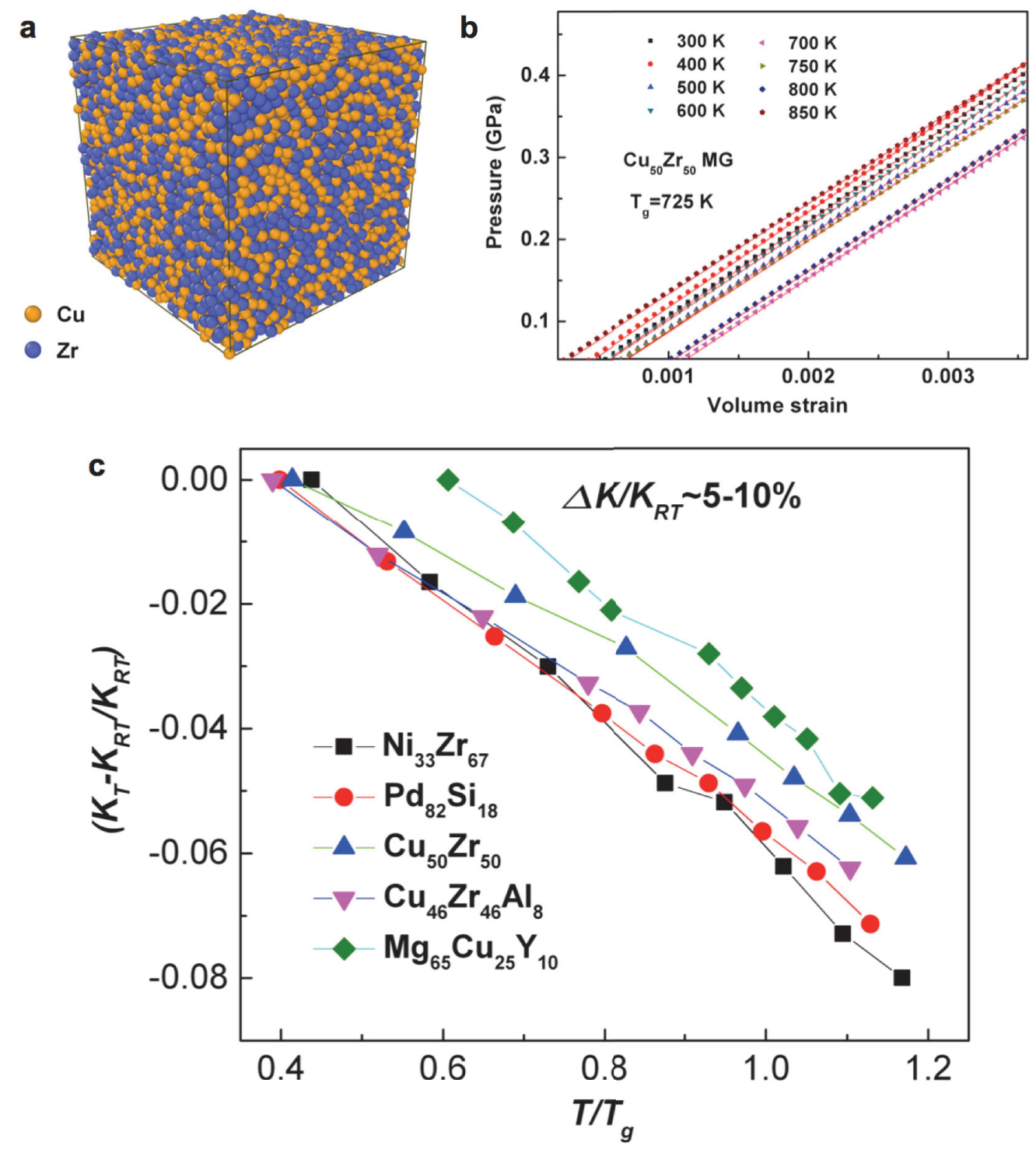

Figure 6: (a). The atomic configuration of $\mathrm{Cu}_{50} \mathrm{Zr}_{50}$ MG sample used in MD simulation. (b) The pressure-volume strain plot for $\mathrm{Cu}_{50} \mathrm{Zr}_{50} \mathrm{MG}$ at different temperatures, from which the bulk modulus can be determined. (c) The relative change of bulk modulus $K$ from room temperature to well above $T_{g}$ for various MGs. 


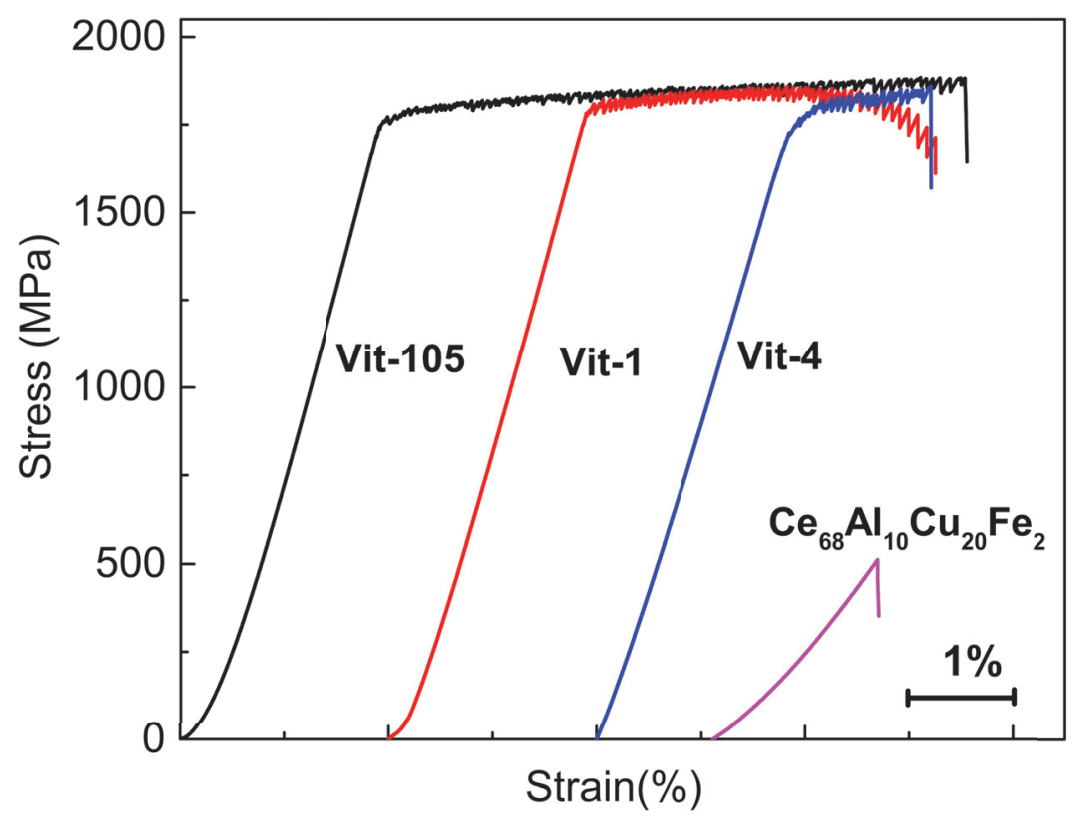

Figure 7: Engineering stress-strain curves for different MGs at the constant strain rate $5 \times 10^{-4} \mathrm{~s}^{-1}$.

$V_{f 0}$ is the initial volume fraction of LLRs in the as-cast state of MGs. This equation can well capture the experimentally determined $V_{f}$ at different annealing times (see Fig. 8) from the measured shear modulus and the relation $V_{f}=\left(G_{0} / G-1\right) / B(\psi)$, where $\psi$ take the values listed in Table 2. The fitted values of $k$ and $n$ are the same as those obtained by fitting elastic moduli data to Eq.5. The form of $V_{f}(t)$ is similar to Johnson-Mehl-Avrami-Kohnogorov (JMAK) equation[53], which usually describes the kinetics of isothermal solid-state transformation, such as the precipitation of a crystalline phase from an amorphous matrix. The difference lies that the evolution of LLRs upon annealing is an annihilation process rather than a growth process, therefore $V_{f}$ decreases monotonically with time. In addition, the fitted values of the exponent, $n$, is in the range $0-1$, which is also smaller than those reported for a crystallization $\operatorname{process}(n \sim 3-4)[54]$ in a three dimensional amorphous structure. In the following, we will show that the stretched exponential form of $V_{f}$ with an exponent $n<1$ reflects the spatial heterogeneity of annihilation dynamics in LLRs during annealing. To understand this, let us first consider the annihilation of LLRs by the reduction on their sizes. Suppose that the total number of LLRs in the unit volume of a MG sample is $N$, 
and the radius of these regions are not uniform and gradually reduces with annealing, the volume fraction of LLRs (equal to the total volume of LLRs here) at the time $t$ can be thus derived as:

$$
V_{f}=\sum_{i=1}^{N} \frac{4}{3} \pi r_{i}^{3}
$$

where $r_{i}$ is the radius of the $i$ th LLR at the time $t$. Denoting $r_{i 0}$ as the initial radius of the $i$ th LLR in the as-cast state, the initial volume fraction of LLRs is $V_{f 0}=\sum_{i=1}^{N}(4 / 3) \pi r_{i 0}^{3}$. Upon annealing, LLRs are gradually annihilated or reduced in their sizes. For simplicity, the size reduction rate of the $i$ th $L L R, d r_{i} / d t$, is proportional to the remaining size, $d r_{i} / d t=-\left(r / \tau_{i}\right)$ with $\tau_{i}$ being a characteristic time scale for the $i$ th LLR. After integration, we obtain: $r_{i}=r_{i 0} \exp \left(-t / \tau_{i}\right)$. Taking this into Eq.7, the variation of the LLR volume fraction with the time can be obtained $V_{f}=\sum_{i=1}^{N}(4 / 3) \pi r_{i 0}^{3} \cdot \exp \left(-3 t / \tau_{i}\right)$. If the annihilation dynamics is uniform, i.e. the values of $\tau_{i}$ are the same for all LLRs(denoted as $\tau_{0}$ ), the volume fraction $V_{f}$ takes on the exponential form: $V_{f}=\sum_{i=1}^{N}(4 / 3) \pi r_{i 0}^{3} \cdot \exp \left(-3 t / \tau_{0}\right)=V_{f 0} \exp \left(-3 t / \tau_{0}\right)$. The derivation of $V_{f}$ can be generalized to the case where LLRs have non-uniform values of $\tau_{i}$ by analogy to the study of dynamic heterogeneity in the primary relaxation process during glass transition[55]. In general, the glass relaxation dynamics follows a exponential equation if there is only a single relaxation time in the relaxation process. However, if there is some distribution of the relaxation time, the stretched exponential equation (the KohlrauschWilliams-Watts(KWW) equation) [55] has to be adopted to describe the relaxation dynamics. Similarly, for non-uniform annihilation dynamics of LLRs, the form of $V_{f}$ should be modified to $V_{f}=V_{f 0} \exp \left[-\left(3 t / \tau_{0}\right)^{n}\right]=V_{f 0} \exp \left(-k t^{n}\right)$ with $k=\left(3 / \tau_{0}\right)^{n}$. The value of the exponent $n$ reflects the distribution of the characteristic time scale, $\tau_{i}$, among LLR. Here, $n=1$ indicates an uniform annihilation time scale, while $n<1$ suggested a distribution of the annihilation time scales in LLRs. In other words, the deviation of $n$ from 1 is indicative of the spatial heterogeneity of LLR annihilation dynamics in MGs.

The established link between elastic properties and structural heterogeneity in MGs is helpful to understand many important correlations between elastic modulus and other prop- 


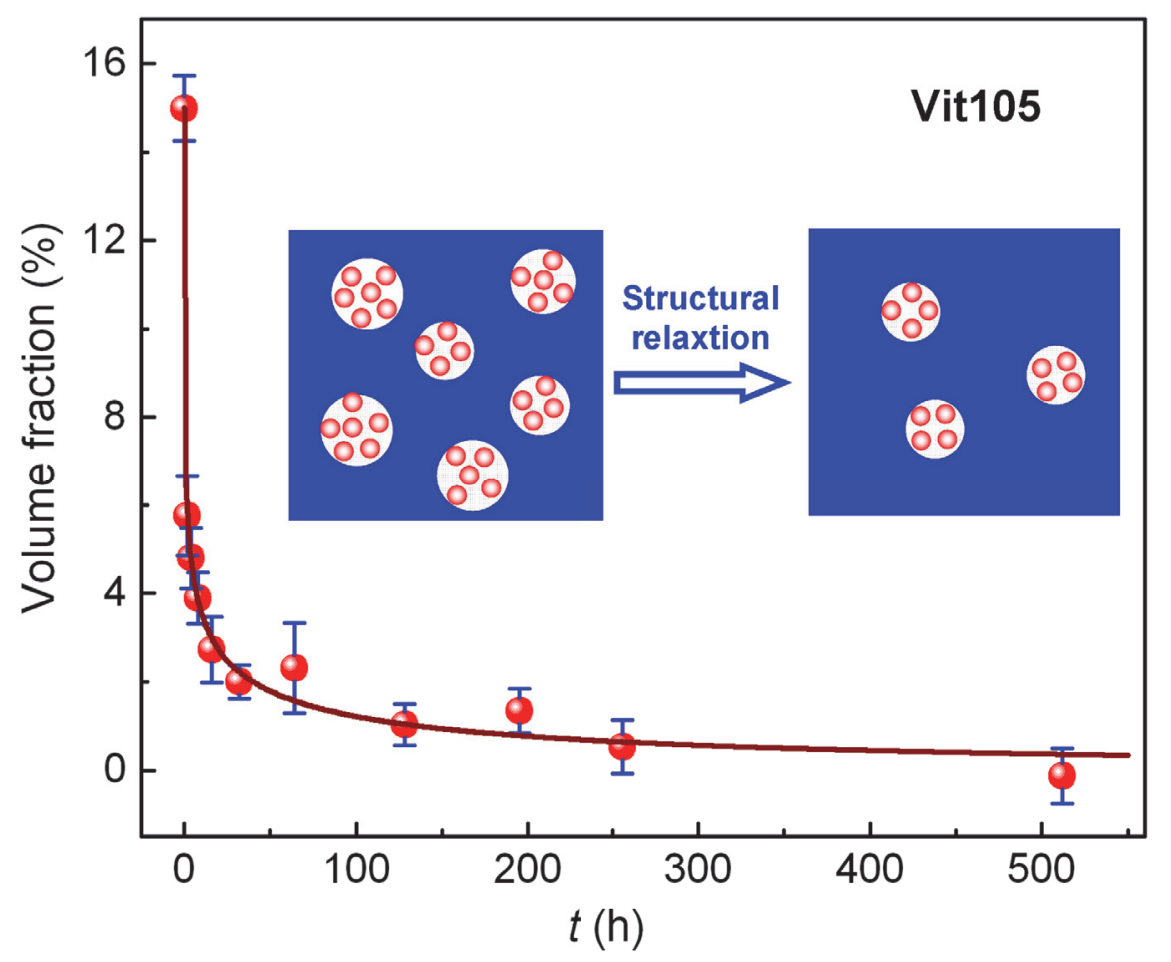

Figure 8: The calculated volume fraction of LLRs, $V_{f}$, versus with the annealing time $t$ for Vit105 based on the measured shear modulus $G$. The upper limit value of $\psi$ was used in the calculation. The inset schematic diagram illustrates the evolution of LLRs with time during structural relaxation. 
erties reported in MGs. For example, the Poisson's ratio was shown to correlate well with the ductility or plasticity in MGs[28], i.e. a larger plasticity tends to favor a higher Poisson's ratio. Now, the correlation can be well understood from our analysis. From Eq. 4, one can see that a higher Poisson's ratio corresponds to a more significant structural heterogeneities of MGs, i.e. a larger volume fraction and/or a greater shear modulus softening of in LLRs. These factors are known to be beneficial to plasticity in MGs. It is also worth noting that the Poisson's ratio criterion for plasticity cannot be directly applied to the MGs (such as the Ce- and La-based MG in our present work) where the bulk modulus softening is much more significant than shear modulus softening in LLRs. The pressure-dominated softening often leads to the increase of Poisson's ratio with the reduction of the LLR volume fraction. Thus, for MGs with pressure-dominated softening, the high value of Poisson's ratio may not promote the plasticity. This may explain that the anomalous brittle behavior for some MGs (such as Au-based MGs[56]) even they have a value of Poisson's ratio. Interestingly, the Poisson's ratio of Au-based MGs almost kept invariant with the thermal annealing[26], which agrees with the case of $\xi=\psi$. In other words, pressure-dominated softening cannot be neglected in these MGs, which explains the unexpected brittleness in the presence of a large Poisson's ratio.

In addition, our present results could also be used to interpret the correlation between the Poisson's ratio in MGs and the fragility of their corresponding glass-forming liquids[24]. It was once shown that a larger Possion's ratio or the ratio of shear modulus to bulk modulus in a glass often corresponds to a more fragile behavior of the corresponding liquid. Novikov et al[24]interpreted the correlation using a non-ergodicity parameter, which determines the amplitude of structural fluctuations frozen at $T_{g}$ and thus relates the fast relaxation dynamics of a liquid to the ratio of shear modulus to bulk modulus in the glass state. Recent experimental studies[57] found that the fragility parameter, $m$, reflects the configuration change rate over the potential energy landscape of a liquid, and thus directly related to the temperature dependence of shear modulus softening around the glass transition. A collection of available experimental data on different kind of glasses[21, 57] also showed that the larger shear modulus softening rate at $T_{g}$ corresponds to the more fragile liquid or the larger 
value of $m$. Since shear softening stems from LLRs according to the present work, it can be then inferred that liquid fragility, as already being found to correlate with the shear softening, should be also correlated with the presence of LLRs or the Poisson?s ratio in general, as reported in Ref.[24]. Provided that the volume fraction of LLRs is fixed, a significant shear modulus softening in LLRs indicates a large value of Poisson's ratio from our current analysis. As a consequence, the fragility of a liquid can be linked to the Poisson's ratio of the glass solids through the shear softening in LLRs or "residual liquid regions" contained in the solid.

\section{Summaries and Conclusions}

In summary, we established a quantitative link between overall elastic properties and local structural heterogeneities in MGs. General formulas for elastic moduli were derived from characteristics of structural hetergeneities from the Eshelby's theory. Our theoretical modeling is verified by the experimental data obtained from various MGs annealed to different degrees. Particularly, we revealed that upon annealing, the Poisson's ratio decreases upon annealing of MGs if the shear softening dominates the behavior of the local soft regions, or increases if pressure softening becomes dominant. The degrees of the bulk and shear modulus softening in local soft regions are extracted for different MGs, and is found to closely correlate with their ductility. The aging dynamics during the annealing process is also quantitatively studied based on the relations between the local elastic heterogeneities and overall elastic constant properties. The implication of our results were also discussed with regard to the Poisson's ratio effect on the ductility and liquid fragility of MGs. Given the generality of Eshelby's theory, the intrinsic correlations herein discovered should be applicable to a wide range of elastically heterogeneous solids.

\section{Acknowledgments}

The work is supported by the Research Grant Council (RGC) of the Hong Kong government through the General Research Fund (GRF) with the account numbers CityU117612, 
11209314, 11207215, 11214914 and the National Science Foundation (NSF) of China (51271195, 51671121, 51601002) and MOST 973 Program (No. 2015CB856800).

\section{Appendix A. Theoretical derivation of elastic properties from structural hetero- geneities in MGs}

Considering an elastic isotropic specimen with a unit volume free of inhomogeneities, the elastic energy for the specimen under a uniform external strain field $\epsilon_{i j}^{A}$ or stress field $\sigma_{i j}^{A}$ is

$$
E_{0}=\frac{1}{2}\left[K_{0} \epsilon^{A} \epsilon^{A}+2 G_{0}\left({ }^{\prime} \epsilon_{i j}^{A}\right)\left({ }^{\prime} \epsilon_{i j}^{A}\right)\right]=\frac{1}{2}\left[\frac{\sigma^{A} \sigma^{A}}{9 K_{0}}+\frac{\left({ }^{\prime} \sigma_{i j}^{A}\right)\left({ }^{\prime} \sigma_{i j}^{A}\right)}{2 G_{0}}\right]
$$

where $K_{0}$ and $G_{0}$ are bulk modulus and shear modulus of the specimen, respectively. $\epsilon^{A}=\epsilon_{m m},{ }^{\prime} \epsilon_{i j}^{A}=\epsilon_{i j}^{A}-(1 / 3) \epsilon_{m m}^{A} \delta_{i j}$, is the deviatoric part of $\epsilon_{i j}^{A}$. Now let us introduce some elastic imhomogeneities into the specimens and meanwhile keep external strain field $\epsilon_{i j}^{A}$. unchanged (i.e. the materials is strained by rigid grips), the interaction between the applied stress and elastic inhomogeneities will cause the increase of total elastic energy in the specimen. According to Eshelby[36], the interaction elastic energy associated with the an elastic inhomogeneity is:

$$
E_{\text {int }}=\frac{1}{2} \int_{V}\left[K_{0} \epsilon^{A} \delta_{i j}+2 G_{0}\left({ }^{\prime} \epsilon_{i j}^{A}\right)\right] \epsilon_{i j}^{T} d V=\frac{1}{2} V \sigma_{i j}^{A} \epsilon_{i j}^{T}
$$

where $V$ is the volume of the inhomogeneity, $\sigma_{i j}^{A}$ is the external stress field which relates with $\epsilon_{i j}^{A}$ by Hook's law. $\epsilon_{i j}^{T}$ is the "equivalent transformation strain" of the inhomogeneity by viewing the inhomogeneity as an Eshelby's inclusion under external applied stress field, and is defined as[36]:

$$
\begin{gathered}
K_{1}\left(\epsilon^{C}+\epsilon^{A}\right)=K_{0}\left(\epsilon^{C}+\epsilon^{A}-\epsilon^{T}\right) \\
G_{1}\left({ }^{\prime} \epsilon_{C}^{i j}+{ }^{\prime} \epsilon_{A}^{i j}\right)=G_{0}\left({ }^{\prime} \epsilon_{i j}^{C}+{ }^{\prime} \epsilon_{i j}^{A}-^{\prime} \epsilon_{i j}^{T}\right)
\end{gathered}
$$

where $\epsilon_{i j}^{C}$ is the strain field within the inhomogeneity caused by the constrain of the matrix on the inhomogeneity. For a spherical inhomogeneity, $\epsilon_{i j}^{C}$ relates with $\epsilon_{i j}^{T}$ by[58]:

$$
\epsilon_{i j}^{C}=S_{i j k l} \epsilon_{k l}^{T}=\left[\frac{5 \nu-1}{15(1-\nu)} \delta_{i j} \delta_{k l}+\frac{4-5 \nu}{15(1-\nu)}\left(\delta_{i k} \delta_{j l}+\delta_{i l} \delta_{j k}\right)\right]
$$


From Eq. A4, we obtain:

$$
\epsilon^{C}=\alpha \epsilon^{T}, \quad{ }^{\prime} \epsilon_{i j}^{C}=\beta^{\prime} \epsilon_{i j}^{T}
$$

where $\alpha=(1 / 3)(1+\nu) /(1-\nu), \beta=(2 / 15)(4-5 \nu) /(1-\nu)$

Combining Eq. A3 into Eq. A5, yields:

$$
\epsilon^{T}=A \epsilon^{A}, \quad{ }^{\prime} \epsilon_{i j}^{T}={ }^{\prime} \epsilon_{i j}^{A}
$$

and $A$ and $B$ are expressed as:

$$
A=\frac{K_{1}-K_{0}}{\left(K_{0}-K_{1}\right) \alpha-K_{0}}, \quad B=\frac{G_{1}-G_{0}}{\left(G_{0}-G_{1}\right) \beta-K_{0}}
$$

Finally, the interaction energy of an inhomogeneity $E_{\text {int }}$ is :

$$
E_{\text {int }}=\frac{1}{2}\left[\frac{A \sigma^{A} \sigma^{A}}{9 K_{0}}+\frac{B\left({ }^{\prime} \sigma_{i j}^{A}\right)\left({ }^{\prime} \sigma_{i j}^{A}\right)}{2 G_{0}}\right]
$$

Thus, the total energy of the system containing a volume fraction $V_{f}$ of spherical inhomogneities is:

$$
E_{\text {total }}=\frac{1}{2}\left[\frac{1+A V_{f}}{9 K_{0}} \sigma^{A} \sigma^{A}+\frac{1+B V_{f}}{2 G_{0}}\left({ }^{\prime} \sigma_{i j}^{A}\right)\left({ }^{\prime} \sigma_{i j}^{A}\right)\right]
$$

Compared Eq. A9 with Eq. A1, one can readily obtain the overall or effective elastic modulus:

$$
K=\frac{K_{0}}{1+A V_{f}}, \quad G=\frac{G_{0}}{1+B V_{f}}
$$

Finally, the effective Poisson's ratio is found to be:

$$
\nu=\frac{3 K-2 G}{6 K+2 G}=\frac{\left(1+\nu_{0}\right)\left(1+B V_{f}\right)-\left(1-2 \nu_{0}\right)\left(1+A V_{f}\right)}{2\left(1+\nu_{0}\right)\left(1+B V_{f}\right)+\left(1-2 \nu_{0}\right)\left(1+A V_{f}\right)}
$$

\section{References}

[1] M. Chen, Mechanical behavior of metallic glasses: Microscopic understanding of strength and ductility, Annu. Rev. Mater. Res. 38 (2008) 445-469.

[2] E. Ma, Tuning order in disorder, Nat Mater 14 (2015) 547-552.

[3] J. Schroers, Bulk metallic glasses, Phys.Today 66 (2013) 32.

[4] K. Yoshimoto, T. S. Jain, K. V. Workum, P. F. Nealey, J. J. de Pablo, Mechanical heterogeneities in model polymer glasses at small length scales, Phys. Rev. Lett. 93 (2004) 175501. 
[5] M. Tsamados, A. Tanguy, C. Goldenberg, J.-L. Barrat, Local elasticity map and plasticity in a model lennard-jones glass, Phys. Rev. E 80 (2009) 026112.

[6] C. Patrick Royall, S. R. Williams, T. Ohtsuka, H. Tanaka, Direct observation of a local structural mechanism for dynamic arrest, Nat Mater 7 (2008) 556-561.

[7] J. Ding, S. Patinet, M. Falk, Y. Cheng, E. Ma, Soft spots and their structural signature in a metallic glass, PNAS 111 (2014) 14052-14056.

[8] Y. Cheng, E. Ma, Atomic-level structure and structure-property relationship in metallic glasses, Prog. Mater. Sci. 56 (2011) $379-473$.

[9] H. Mizuno, S. Mossa, J.-L. Barrat, Measuring spatial distribution of the local elastic modulus in glasses, Phys. Rev. E 87 (2013) 042306.

[10] W. Dmowski, T. Iwashita, C.-P. Chuang, J. Almer, T. Egami, Elastic heterogeneity in metallic glasses, Phys. Rev. Lett. 105 (2010) 205502.

[11] Y. H. Liu, D. Wang, K. Nakajima, W. Zhang, A. Hirata, T. Nishi, A. Inoue, M. W. Chen, Characterization of nanoscale mechanical heterogeneity in a metallic glass by dynamic force microscopy, Phys. Rev. Lett. 106 (2011) 125504.

[12] H. Wagner, D. Bedorf, S. Küchemann, M. Schwabe, B. Zhang, W. Arnold, K. Samwer, Local elastic properties of a metallic glass, Nat Mater 10 (2011) 439-442.

[13] J. C. Ye, J. Lu, C. T. Liu, Q. Wang, Y. Yang, Atomistic free-volume zones and inelastic deformation of metallic glasses, Nat Mater 9 (2010) 619-623.

[14] V. Mazzacurati, G. Ruocco, M. Sampoli, Low-frequency atomic motion in a model glass, EPL 34 (1996) 681

[15] H. R. Schober, C. Oligschleger, Low-frequency vibrations in a model glass, Phys. Rev. B 53 (1996) 11469-11480.

[16] W. Jiao, B. A. Sun, P. Wen, H. Y. Bai, Q. P. Kong, W. H. Wang, Crossover from stochastic activation to cooperative motions of shear transformation zones in metallic glasses, App. Phys. Lett. 103 (2013).

[17] H. Ke, J. Zeng, C. Liu, Y. Yang, Structure heterogeneity in metallic glass: Modeling and experiment, J. Mater. Sci. Tech. 30 (2014) $560-565$.

[18] J.-O. Krisponeit, S. Pitikaris, K. E. Avila, S. Küchemann, A. Krüger, K. Samwer, Crossover from random three-dimensional avalanches to correlated nano shear bands in metallic glasses, Nat Commun $5(2014)$.

[19] Z. Wang, B. A. Sun, H. Y. Bai, W. H. Wang, Evolution of hidden localized flow during glass-to-liquid transition in metallic glass, Nat Commun 5 (2014).

[20] Q. Wang, S. T. Zhang, Y. Yang, Y. D. Dong, C. T. Liu, J. Lu, Unusual fast secondary relaxation in metallic glass, Nat Commun 6 (2015). 
[21] W. H. Wang, The elastic properties, elastic models and elastic perspectives of metallic glasses, Progress in Materials Science 57 (2012) 487 - 656.

[22] B. Sun, W. Wang, The fracture of bulk metallic glasses, Prog. Mater. Sci. 74 (2015) $211-307$.

[23] J. C. Dyre, Colloquium: The glass transition and elastic models of glass-forming liquids, Rev. Mod. Phys. 78 (2006) 953-972.

[24] V. N. Novikov, A. P. Sokolov, Poisson's ratio and the fragility of glass-forming liquids, Nature 431 (2004) 961-963.

[25] T. Egami, Formation and deformation of metallic glasses: Atomistic theory, Intermetallics 14 (2006) $882-887$.

[26] Z. Evenson, S. E. Naleway, S. Wei, O. Gross, J. J. Kruzic, I. Gallino, W. Possart, M. Stommel, R. Busch, $\beta$ relaxation and low-temperature aging in a au-based bulk metallic glass: From elastic properties to atomic-scale structure, Phys. Rev. B 89 (2014) 174204.

[27] L. Huo, J. Zeng, W. Wang, C. Liu, Y. Yang, The dependence of shear modulus on dynamic relaxation and evolution of local structural heterogeneity in a metallic glass, Acta Materialia 61 (2013) 4329 4338.

[28] J. J. Lewandowski, W. H. Wang, A. L. Greer, Intrinsic plasticity or brittleness of metallic glasses, Philo. Mag. Lett. 85 (2005) 77-87.

[29] K. L. Ngai, L.-M. Wang, R. Liu, W. H. Wang, Microscopic dynamics perspective on the relationship between poisson's ratio and ductility of metallic glasses, J. Phys.Chem. 140 (2014).

[30] Y. Q. Cheng, E. Ma, Configurational dependence of elastic modulus of metallic glass, Phys. Rev. B 80 (2009) 064104.

[31] H. S. Arora, A. V. Aditya, S. Mukherjee, Structural relaxation driven increase in elastic modulus for a bulk metallic glass, J. App. Phys. 117 (2015).

[32] A. Kursumović, M. Scott, E. Girt, R. Cahn, Changes in the young's modulus during structural relaxation of a metallic glass, Scrip. Metall. 14 (1980) $1303-1308$.

[33] S. V. Ketov, Y. H. Sun, S. Nachum, Z. Lu, A. Checchi, A. R. Beraldin, H. Y. Bai, W. H. Wang, D. V. Louzguine-Luzgin, M. A. Carpenter, A. L. Greer, Rejuvenation of metallic glasses by non-affine thermal strain, Nature 524 (2015) 200-203.

[34] Y. Tong, T. Iwashita, W. Dmowski, H. Bei, Y. Yokoyama, T. Egami, Structural rejuvenation in bulk metallic glasses, Acta Mater. 86 (2015) 240 - 246.

[35] S. Liu, W. Jiao, B. Sun, W. Wang, A quasi-phase perspective on flow units of glass transition and plastic flow in metallic glasses, J. Non-Crys. Solids 376 (2013) $76-80$.

[36] J. D. Eshelby, The determination of the elastic field of an ellipsoidal inclusion, and related problems, Proc. R. Soc. London, Ser. A 241 (1957) 376-396. 
[37] J. S. Langer, Microstructural shear localization in plastic deformation of amorphous solids, Phys. Rev. E 64 (2001) 011504.

[38] C. Maloney, A. Lemaître, Subextensive scaling in the athermal, quasistatic limit of amorphous matter in plastic shear flow, Phys. Rev. Lett. 93 (2004) 016001.

[39] R. Dasgupta, H. G. E. Hentschel, I. Procaccia, Microscopic mechanism of shear bands in amorphous solids, Phys. Rev. Lett. 109 (2012) 255502.

[40] G. N. Greaves, A. L. Greer, R. S. Lakes, T. Rouxel, Poisson's ratio and modern materials, Nat Mater 10 (2011) 823-837.

[41] W. Li, Y. Gao, H. Bei, On the correlation between microscopic structural heterogeneity and embrittlement behavior in metallic glasses, Sci. Rep. 5 (2015) 14786 EP -.

[42] P. Wen, G. P. Johari, R. J. Wang, W. H. Wang, Change in the vibrational properties of bulk metal glass with time, Phys. Rev. B 73 (2006) 224203.

[43] P. Murali, U. Ramamurty, Embrittlement of a bulk metallic glass due to sub-tg annealing, Acta Materialia 53 (2005) 1467 - 1478.

[44] S. V. Nemilov, G. P. Johari, A mechanism for spontaneous relaxation of glass at room temperature, Philo. Mag. 83 (2003) 3117-3132.

[45] R. Davies, G. Jones, Thermodynamic and kinetic properties of glasses, Adv. Phys 2 (1953) 370-410.

[46] B. A. Sun, Z. Y. Liu, Y. Yang, C. T. Liu, Delayed shear banding and evolution of local plastic flow in a metallic glass, Applied Physics Letters 105 (2014).

[47] Y. Yang, C. T. Liu, Size effect on stability of shear-band propagation in bulk metallic glasses: an overview, Journal of Materials Science 47 (2012) 55-67.

[48] B. A. Sun, H. B. Yu, W. Jiao, H. Y. Bai, D. Q. Zhao, W. H. Wang, Plasticity of ductile metallic glasses: A self-organized critical state, Phys. Rev. Lett. 105 (2010) 035501.

[49] H. Ke, B. Sun, C. Liu, Y. Yang, Effect of size and base-element on the jerky flow dynamics in metallic glass, Acta Materialia 63 (2014) $180-190$.

[50] R. Maass, D. Klaum'unzer, J. L'offler, Propagation dynamics of individual shear bands during inhomogeneous flow in a zr-based bulk metallic glass, Acta Materialia 59 (2011) 3205 - 3213.

[51] B. A. Sun, Y. Yang, W. H. Wang, C. T. Liu, The critical criterion on runaway shear banding in metallic glasses, Scientific Reports 6 (2016) 21388.

[52] A. Greer, Y. Cheng, E. Ma, Shear bands in metallic glasses, Mater. Sci. Eng: R 74 (2013) $71-132$.

[53] M. Fanfoni, M. Tomellini, The johnson-mehl- avrami-kohnogorov model: A brief review, Il Nuovo Cimento D 20 (1998) 1171-1182.

[54] J. Málek, Calorimetry and thermal analysis the applicability of johnson-mehl-avrami model in the thermal analysis of the crystallization kinetics of glasses, Thermo. Acta 267 (1995) $61-73$. 
[55] M. D. Ediger*, C. A. Angell*, S. R. Nagel*, Supercooled liquids and glasses, The Journal of Physical Chemistry 100 (1996) 13200-13212.

[56] S. Madge, D. Louzguine-Luzgin, J. Lewandowski, A. Greer, Toughness, extrinsic effects and poisson's ratio of bulk metallic glasses, Acta Materialia 60 (2012) 4800 - 4809.

[57] B. Zhang, H. Y. Bai, R. J. Wang, Y. Wu, W. H. Wang, Shear modulus as a dominant parameter in glass transitions: Ultrasonic measurement of the temperature dependence of elastic properties of glasses, Phys. Rev. B 76 (2007) 012201.

[58] R. Dasgupta, O. Gendelman, P. Mishra, I. Procaccia, C. A. B. Z. Shor, Shear localization in threedimensional amorphous solids, Phys. Rev. E 88 (2013) 032401. 